\title{
Situação epidemiológica da tuberculose infantil no Município do Rio de J aneiro
}

\author{
Epidemiological analysis of pediatric tuberculosis \\ in the city of Rio de J aneiro
}

Henriqueta Maria Valporto Oliveira 1

Antonio Ruffino-Netto 2

Guida Silva Vasconcellos 1

Selma Maria Oliveira Dias 1

1 Secretaria Municipal de Saúde do Rio de Janeiro. Rua Afonso Caval canti 455, Rio de Janeiro, 20211-110, RJ.

2 Faculdade de Medicina de Ribeirão Preto, Universidade de São Paulo.

Av. Bandei rantes 3900,

Ribeirão Preto, 14049-900, SP.
Abstract With the purpose of studying the epidemiological situation of pediatric tuberculosis in Rio de Janeiro, we analyze the reporting forms for 560 pati ents under 15 years of age, among those being monitored by the city Tuberculosis Control Program. The foll owing variables were studied: age, clinical form, results of laboratory tests, and places where the patients were seen. About $96 \%$ of the data investigated was found. The main results were the following: 1 . the relative percentage of cases among children compared to adults was similar in Rio de Janeiro (6.6\%) and Brazil (6.7\%); both were far bel ow the percentage expected by the Brazilian Ministry of Health; $2.70 \%$ of cases presented with the pulmonary form of the di sease; among these, microscopy was performed in $50 \%$, with $29 \%$ of positive results; $38 \%$ of the patients displayed radiological lesions, with signs of cavitation; 3 . only $24 \%$ of the case contacts were controlled; 4 . incidence rates of all forms of tuberculosis in Rio de Janeiro were much higher (as much as 62.6 times higher for some coefficients) as compared to Brazil as a whole; and 5. prevalence of positiveserology for HIV was at least $4.1 \%$.

Key words Tuberculosis; Child Health; Epidemiology; HIV

Resumo O trabalho propõe-se estudar a situação epidemiológica da tuberculose infantil no Município do Rio de Janeiro. Foram analisadas 560 fichas de notificação dos paciente de zero a 14 anos, inscritos no Programa de Controle da Tuberculose do Município, em 1993. Com base nas variáveis: idade, forma clínica da doença, exames complementares, local de atendimento, encontramos pel o menos 96,1\% das informações almejadas. Foi possível observar: 1) o percentual relativo casos infância/adultos é semel hante entre Rio de Janeiro (6,6\%) e Brasil (6,7\%); tais valores são bem abaixo do valor apregoado pelo Ministério da Saúde; 2) setenta por cento dos casos são da forma pulmonar; destes, em 50\% foi efetuada a baciloscopia, encontrando-se $29 \%$ posi ti vos; 38\% apresentavam lesões radiol ógi cas com sinais de cavitação; 3 ) apenas $24 \%$ dos comunicantes foram controlados; 4) os coefici entes de inci dência de todas as formas da doença são muitas vezes superior (chegando a 62,6 vezes para certos coeficientes) em relação ao Brasil; 5) a prevalência de sorologia positiva para HIV é de pelo menos 4,1\%.

Palavras chave Tuberculose; Saúdelnfantil; Epidemiologia; HIV 


\section{Introdução}

Pio (1984) refletindo sobre o futuro da luta antituberculose, levanta as questões: Seria realista a estratégia mundial de saúde para todos no ano 2000 no que se refere ao controle da tuberculose? O que se teria alcançado no ano 2000 ?

Dentro da reflexão, assinala que, no final da década de 40 , a disponibilidade de vacinação com BCG, Raios X em massa e quimioterapia específica criou um otimismo exagerado com respeito ao controle da tuberculose. Previsões foram feitas de que a tuberculose deixaria de ser ameaça para a saúde pública por volta de 1975. Isto não ocorreu. Por ocasião do princípio da década de 60 , julgou-se que o problema não era tanto de natureza técnica e sim administrativa, ou seja, os instrumentos técnicos de controle eram todos conhecidos, o que se necessitava era modificar a estrutura dos serviços especial izados, acabar com campanhas e conceber-se um programa integrado de luta antituberculose. Exame efetuado pela OMS e UICT no início da década de 80 indicava que a situação epidemiológica da endemia no mundo tinha se estabilizado; enquanto em al guns países a incidência vinha diminuindo, na grande maioria daqueles em desenvolvimento o risco de infecção continuava alto. Decorridos vinte anos de formulação de política integrada de programas de controle da doença não se obteve a redução esperada. Sinal de fracasso dos meios técnicos? Ou seriam os nossos pressupostos que estariam errados? Para a esperada integração dos programas de controle da tuberculose aos serviços gerais de saúde, valeria a pena fazermos as perguntas: integrar o que com quem? onde estão os serviços de saúde a serem integrados? Nos países do terceiro mundo, serviços gerais de saúde, assim como os de educação, foram totalmente marginalizados. Concordamos com Pio quando ainda assinala "tuberculose é um componente bastante pequeno do problema de saúde e este, por sua vez, é só um entre os muitos problemas sociais, como explosão demográfica, a fome, o analfabetismo, a falta de empregos e moradias".

E a tuberculose aí está, no mundo, matando sozinha mais que o conjunto de todas as outras moléstias infecto-contagiosas somadas.

No nível mundial, estimam-se 1 bilhão e 700 milhões de pessoas infectadas pelo M. tuberculosis, isto é, um terço da população. Dados referentes ao ano de 1988 mostram que são diagnosticados anualmente de oito a dez miIhões de casos novos da doença, dos quais cerca de três milhões vão ao óbito (WHO, 1989).
Assim, a endemia da doença, que estava caminhando para certo controle em alguns países, "descontrolada " ainda em outros, tem sua tendência marcantemente alterada frente $o$ advento da Aids. Esta última tem sido apontada como a mais significativa ocorrência que alterou o "equilibrio entre o homem e o BK nos últimos cem 100 anos, sendo decisiva no risco de adoecimento por tuberculose, seja por reativação endógena ou infecção exógena" (MS, 1994).

Atualmente no Brasil, o número de notificações é da ordem de noventa mil casos anuais (se consideraramos que isto significa apenas de $75 \%$ a $80 \%$ da verdadei ra incidência, o número real de casos deverá ser algo entre 112.500 a 120.000). Em termos de notificação anual, estão na frente do Brasil apenas Índia, Indonésia, China, Filipinas e Paquistão.

O Estado do Rio de Janeiro em 1992 notificou 15.858 casos. Evidentemente a Aids (entre outros fatores) tem contribuído muito com o aumento desta incidência. A situação da tuberculose infantil neste contexto torna-se muito complicada por diferentes razões: seja por tratarem-se de crianças desnutridas," abandonadas", seja por razões ligadas ao diagnóstico propriamente dito (como é sabido, difícil neste grupo etário). Assim é que muitas crianças têm chegado aos serviços de saúde com quadros patológicos avançados. Em vista desta problemática, em 1992 juntaram-se 120 profissionais para realizar o Fórum sobre Tuberculose Infantil, a fim de, em comum acordo, Estado e Município do Rio de Janeiro tentarem traçar diretrizes para o controle do referido problema. Ao final do evento, enviaram as diretrizes às autoridades governamentais competentes.

Foram propostas fundamentais deste fórum: 1) treinamento de pediatras e clínicos que atuam no programa de controle da tuberculose (PCT), lotados na rede municipal de saúde para o diagnóstico e tratamento da tuberculose infantil; 2) implementação do diagnóstico e tratamento da tuberculose infantil através da capacitação dos profissionais e supervisão direta nas unidades de saúde; 3) agendamento rigoroso de todos os comunicantes infantis dos casos índice de tuberculose, para que sejam submetidos a exames clínico e laboratorial para a detecção precoce da doença, bem como para a indicação de quimioprofilaxia; 4) estabelecimento de um sistema de referência e contra-referência formal para o diagnóstico e tratamento da tuberculose infantil; 5) sensibilização da população para o problema da tuberculose infantil, através da divulgação de informações pela imprensa escrita e/ ou falada, palestras nas escolas, comunidades, unidades de saúde e instituições. 
Assim, este trabal ho, levando em consideração as diretrizes apontadas anteriormente, buscando aprimorar/reformular/aclarar normas técnicas operacionais para se maximizar o PCT infantil, propõe-se, como primeira modesta contribuição, analisar as características clínicoepidemiológicas da tuberculose infantil no Município do Rio de Janeiro e tentar identificar fatores operacionais que influenciam nos indicadores epidemiológicos encontrados.

\section{Material e métodos}

População de Referência - crianças de zero a 14 anos de idade residentes no Município do Rio de Janeiro, portadoras de tuberculose.

População de Estudo - crianças da população de referência que demandaram os Centros de Saúde e Hospitais do Rio de Janeiro (que desenvolvem PCT); foram diagnosticadas como portadoras de tuberculose e tiveram seus casos notificados, sendo, portanto, inscritas no PCT no nível do Município em 1993.

Participantes - optou-se por não se fazer amostragem e sim estudar toda a população de estudo.

Com base na ficha de notificação dos casos, foram levantadas informações pertinentes às seguintes variáveis: idade, sexo, forma clínica da doença, exames complementares (baciloscopia, Raios X, sorologia para pesquisa de HIV), local de atendimento, município de procedência.

\section{Resultados e discussão}

Em 1993, foram notificados 8.484 casos de tuberculose no Município do Rio de Janeiro, sendo que em $85 \%$ (7.178 ) o atendimento/ notificação foi efetuado pelos Centros Municipais de Saúde e em $15 \%$ (1.268) foi pelos Hospitais. Deste total de 8.484 casos, 560 (6,6\%) ocorreram em crianças de zero a 14 anos de idade. Neste mesmo ano no Brasil foram notificados 80.663 casos, e o número de tuberculosos no grupo etário zero a 14 anos foi 5.435 , isto é, $6,7 \%$ do total (Tabela 1). O percentual de casos notificados nas crianças e/ ou adultos não apresenta diferença estatisticamente significante entre Rio de Janeiro (6,6\%) e Brasil (6,7\%) ( $c 2=0,23 ; p=0,63$ ).

O Ministério da Saúde (1992) assinala que, no País, $15 \%$ dos casos de tuberculose supostamente ocorreriam no grupo etário de zero a 14 anos. Para o Município do Rio de Janeiro, este percentual foi apenas de $6,6 \%$. Seria esta pressuposição realista/ adequada para o País e/ ou região do Rio de Janeiro? Na hipótese de pressuposição correta, que elementos de natureza epidemiológica e/ ou operacional poderiam explicar esta discrepância entre o esperado (15\%) e o observado (6\%)? Várias explicações poderiam ser aventadas.

Em primeiro lugar, o epidemiologista pensaria em erro de informações ligadas ao sistema de vigilância da doença, que não estaria captando com sensibilidade suficiente o que estivesse ocorrendo; operacionalmente, os serviços de saúde não estariam cumprindo adequadamente com suas funções. Mesmo sabendo que os serviços de saúde pública do Rio de Janeiro podem deixar a desejar, a hipótese acima sozinha não poderia explicar a discrepância entre $6,6 \%$ e $15 \%$. Primeiro que o percentual do Rio de Janeiro $(6,6 \%)$ é igual ao do restante do País (6,7\%) e, portanto, a deficiência de serviços seria exatamente igual em todo o território nacional, o que não parece ter muito sentido. Ou por outra, é de se supor que o Rio tenha, pelo menos, mais condições de oferecer melhor serviço em pauta.

Outra hipótese explicativa seria o efeito acumulativo dos programas de vacinação em massa com BCG, que começariam a apresentar sua eficácia, modificando, portanto, a distribuição percentual dos casos de tuberculose como um todo. Favoravelmente a esta hipótese, tem-se que a cobertura vacinal no Município tem-se mantido alta (134\%; 126\%; 134\%; 169\% respectivamente para os anos de 1991; 1992; 1993 e 1994).

Quer nos parecer que um pouco dos diferentes fatores anteriomente levantados (epidemiológicos e/ ou operacionais) poderiam explicar a discrepância, acrescentando-se, ainda, a possível irrealidade da pressuposição ministerial.

Tabela 1

Distribuição de casos notificados de tuberculose segundo local e grupo etário - 1993.

\begin{tabular}{|c|c|c|c|c|}
\hline \multirow[t]{2}{*}{ Grupo etário (em anos) } & \multicolumn{2}{|c|}{ Brasil } & \multicolumn{2}{|c|}{ Rio de J aneiro } \\
\hline & $n$ & $\%$ & $\mathrm{n}$ & $\%$ \\
\hline 0 a 14 & 5.435 & 6,7 & 560 & 6,6 \\
\hline$\geq 15$ & 75.228 & 93,3 & 7.924 & 93,4 \\
\hline Total & 80.663 & 100,0 & 8.484 & 100,00 \\
\hline
\end{tabular}

$c 2=0,23 ; p=0,63$ 
Tabela 2

Distribuição de casos de tuberculose notificados no Município do Rio de J aneiro, em 1993, segundo forma clínica e grupo etário.

\begin{tabular}{|c|c|c|c|c|c|c|c|c|}
\hline \multirow[t]{3}{*}{ Forma clínica } & \multicolumn{6}{|c|}{ Grupo etário (em anos) } & & \\
\hline & \multicolumn{2}{|c|}{$0-4$} & \multicolumn{2}{|c|}{$5-9$} & \multicolumn{2}{|c|}{$10-14$} & \multicolumn{2}{|c|}{ Total } \\
\hline & $\mathrm{n}$ & $\%$ & $\mathrm{n}$ & $\%$ & $n$ & $\%$ & $\mathrm{n}$ & $\%$ \\
\hline Pulmonar & 248 & 72,7 & 57 & 55,9 & 75 & 64,1 & 380 & 67,9 \\
\hline Meningite tuberculosa & 9 & 2,6 & 3 & 2,9 & - & - & 12 & 2,1 \\
\hline Pleural & 13 & 3,8 & 2 & 2,0 & 22 & 18,8 & 37 & 6,6 \\
\hline Ganglionar & 31 & 9,1 & 30 & 29,4 & 9 & 7,7 & 70 & 12,5 \\
\hline Óssea & 9 & 2,6 & 3 & 2,9 & - & - & 12 & 2,1 \\
\hline Gênito-urinária & 1 & 0,3 & - & - & 1 & 0,9 & 2 & 0,4 \\
\hline Miliar & 1 & 0,3 & - & - & - & - & 1 & 0,2 \\
\hline Ocular & 2 & 0,6 & - & - & 1 & 0,9 & 3 & 0,5 \\
\hline Outras & 22 & 6,5 & 5 & 4,9 & 8 & 6,8 & 35 & 6,3 \\
\hline Sem informação & 5 & 1,5 & 2 & 2,0 & 1 & 0,9 & 8 & 1,4 \\
\hline Total & 341 & 100 & 102 & 100 & 117 & 100 & 560 & 100 \\
\hline
\end{tabular}

Na Tabela 2, apresentamos a distribuição dos casos notificados segundo a forma clínica e o grupo etário.

Observamos inicialmente que o percentual de notificações sem informação (da forma clínica) é de apenas $1,4 \%$, o que, de certa maneira, permite um pouco mais de confiança nas análises que se seguem. Em $68 \%$ dos casos, trata-se de forma pulmonar, abaixo dos $75 \%$ que seria esperado pelo Ministério da Saúde (MS, 1992). Esta diferença é estatisticamente significante $(p<0,001)$. Indica que o percentual de formas extrapulmonares está acima do que se espera. Esta discrepância, antes de traduzir qualquer fator de ordem epidemiológica, parece-nos muito mais de ordem operacional que de qualquer outra fonte. $O$ porte da cidade em pauta tem grande quantidade de pediatras especialistas, assim como outros recursos técnicos, que bem poderiam estar detectando (às vezes até com exagero) outras formas de tuberculose que não a pulmonar (lembrar que 15\% dos diagnósticos são feitos em hospitais de referência). Ou seria a explicação ao contrário: apesar do grande número de pediatras, estes, no entanto, estão pouco capacitados para diagnosticar a tuberculose pulmonar? Esta segunda hipótese nos parece a menos provável.

Com freqüência, aparece em segundo lugar a tuberculose ganglionar (13,5\%), seguida da forma pleural $(6,6 \%)$. Apesar da alta cobertura vacinal com BCG (como já mostrado anteriormente), registraram-se 12 casos de meningite tuberculosa no ano de 1993 (em 1991, 1992 e 1994 registraram-se repectivamente 17,17 e 12 casos desta doença).
Tabela 3

Distribuição dos casos notificados de tuberculose em função do resultado da baciloscopia.

\begin{tabular}{lrc}
\hline Baciloscopia & \multicolumn{2}{c}{ Total } \\
& $n$ & $\%$ \\
\hline Positiva & 111 & 29,2 \\
Negativa & 77 & 20,3 \\
Não realizada & 192 & 50,5 \\
Total & 380 & 100 \\
\hline
\end{tabular}

Observa-se ainda que $61 \%$ dos casos notificados estão no grupo etário de zero a quatro anos; $21 \%$ no grupo de dez a 14 e $18 \%$ no de cinco a nove anos. Esta distribuição segue em linhas gerais a curva padrão de morbidade na infância em diferentes partes do mundo. As formas graves de tuberculose (meningite e/ ou miliar) aparecem com freqüência relativamente baixa, $(2,3 \%)$.

Dos 380 casos notificados como tuberculose pulmonar, encontramos que em $50 \%$ foi efetuada a baciloscopia, observando-se que $30 \%$ apresentam baciloscopia ou cultura positiva ( Tabelas 3 e 4). Operacionalmente, isto indica o uso deste instrumento diagnóstico na infância, acrescido do achado do elevado número de casos positivos. Para este grupo etário, esperarse-ia um percentual de $20 \%$ de positividade. Seria este excessivo percentual observado devido à boa qualidade dos hopitais de referência que realizam estes exames? Hipótese bem provável e difícil de ser descartada. Dos 111 casos 
Distribuição dos casos notificados de tuberculose segundo o resultado da baciloscopia.

\begin{tabular}{|c|c|c|c|c|c|c|c|c|}
\hline \multirow[t]{3}{*}{ Resultado da baciloscopia } & \multicolumn{6}{|c|}{ Grupo etário (em anos) } & \multicolumn{2}{|c|}{ Total } \\
\hline & \multicolumn{2}{|c|}{$0-4$} & \multicolumn{2}{|c|}{$5-9$} & \multicolumn{2}{|c|}{$10-14$} & \multirow[b]{2}{*}{$\mathrm{n}$} & \multirow[b]{2}{*}{$\%$} \\
\hline & $n$ & $\%$ & $\mathrm{n}$ & $\%$ & $\mathrm{n}$ & $\%$ & & \\
\hline Positiva + & 25 & 29,8 & 2 & 40 & 10 & 45,5 & 37 & 33,3 \\
\hline Positiva ++ & 34 & 40,5 & - & - & 5 & 22,7 & 39 & 35,1 \\
\hline Positiva +++ & 13 & 15,5 & 2 & 40 & 3 & 13,6 & 18 & 16,2 \\
\hline Positiva sem especificação & 7 & 8,3 & - & - & 1 & 4,5 & 8 & 7,2 \\
\hline Baciloscopia - Cultura+ & 5 & 6,0 & 1 & 20 & 3 & 13,6 & 9 & 8,1 \\
\hline Total & 84 & 100 & 5 & 100 & 22 & 100 & 111 & 100 \\
\hline
\end{tabular}

positivos (à baciloscopia ou cultura), tem-se que 84 estão no grupo etário de zero a quatro anos. Lembrando que neste grupo foram notificados 341 casos, resulta um percentual de positividade de $25 \%$ (84/341); no grupo etário cinco a nove anos, houve apenas cinco casos positivos, isto é, 5\% ( 5/ 102), e no grupo de dez a 14 anos, 22 foram positivos, ou seja, 19\% (22/ 117). Dois aspectos interessantes: enquanto nos abaixo de cinco anos o percentual é bem elevado, entre cinco e nove anos ele é extremamente baixo. Tornar-se-ia difícil tentar compreender somente pela fisiopatologia da doença.

Possivelmente os cuidados envolvidos na operacionalização deste instrumento diagnóstico está sendo diferente em função da idade.

Na Tabela 5, observamos a distribuição dos achados radiológicos nas 380 crianças com tuberculose pulmonar. Em 93,7\% acham-se assinaladas alterações radiológicas, sendo $46,3 \%$ sem referência a cavitações e 37,6\% com cavitações; $2,4 \%$ com Raios $X$ normal e em apenas $3,9 \%$ não obtivemos esta informação. O elevado percentual de casos já com cavitação indicaria um diagnóstico mais tardio associado ao retardo operacional da rede de serviços? Ou seria uma aceleração do processo pertinente ao hospedeiro em si (desnutrição, imunodeficiência etc.)? Todos estes fatores devem ter sua contribuição. Quanto às atividades da rede no controle de comunicantes, deve-se assinalar: de um total de 8.484 casos índices de tuberculose notificados, esperar-se-ia que 33.936 comunicantes fossem examinados (exame de quatro comunicantes para cada caso índice). Contudo, neste ano, foram examinados apenas 8.293 , isto é, $24 \%$ do esperado. Sem dúvida, neste aspecto, a rede está operando aquém do esperado.

Quanto aos fatores ligados ao hospedeiro em si, poder-se-ia lembrar do nível nutricional destas crianças e fundamentalmente da ocorrência de infecção pelo HIV. O levantamento
Tabela 5

Distribuição de achados radiológicos em crianças portadoras de tuberculose pulmonar.

\begin{tabular}{lrc}
\hline Achado radiológico & $\mathrm{n}$ & $\%$ \\
\hline Anormal sem cavitação & 176 & 46,3 \\
Anormal com cavitação & 143 & 37,6 \\
Lesão pleural & 28 & 7,4 \\
Lesão ganglionar & 9 & 2,4 \\
Normal & 9 & 2,4 \\
Sem informação & 15 & 3,9 \\
Total & 380 & 100 \\
\hline
\end{tabular}

efetuado para estimar a soroprevalência de anticorpos anti-HIV obteve o seguinte resultado nas crianças em que o exame foi feito: positivo em 4,1\%; negativo em 4,6\%; sem informação em $3,2 \%$. Em $84,6 \%$ a pesquisa de anticorpos não foi realizada, e em 3,5\% havia indicação clínica para sua realização, porém não efetuada. Isto permite imaginar uma prevalência de pelo menos $4 \%$, variando até possivelmente $7 \%$.

Loures (1995) considerando uma incidência anual de cerca de sessenta mil casos de tuberculose nos adultos, prevê que, por volta do ano 1999 , ocorreriam $25 \%$ a mais de casos da doença devido à epidemia do HIV/ Aids.

Ruffino-Netto (1995) propõe um modelo para avaliar o excesso de casos de tuberculose atribuídos à infecção HIV/ Aids. No modelo citado, simulando uma situação em que a prevalência de anticorpos anti-HIV fosse da ordem de $2 \%$ e risco de infecção de $0,8 \%$, estimou-se (para a população geral e no Brasil atual) um excesso de casos de tuberculose de $17 \%$. Se imaginássemos agora uma prevalência geral da ordem de $4 \%$ (equivalente àquela encontrada no Rio de Janeiro no grupo etário de zero a 14 anos) e um risco de infecção de 1\%, teríamos 
um excesso atual de casos de tuberculose de $36 \%$.

Até este instante, vínhamos tratando a magnitude do problema apenas em termos de números absolutos, isto é, freqüência de ocorrência de eventos. Tais informações são extremamente importantes para o planejador em saúde pública, porém deixam a desejar para o epidemiologista, que está mais interessado nas taxas de ocorrência destes eventos nas populações expostas. Para tanto, são apresentados na Tabela 6 os coeficientes de incidência por cem mil, nos diferentes grupos etários, comparando-se os dados do Município do Rio de Janeiro em 1993 com aqueles observados no Brasil em 1990 (MS, 1993), que são os disponíveis que obtivemos no momento. Inicialmente, chama $a$ atenção que a incidência de tuberculose na população geral do Rio de Janeiro tenha sido 3,16 vezes maior que a observada no Brasil $(152,1 / 48,2)$. As relações: incidências de formas de tuberculose e/ ou categoria (ser ou não bacilífero) do Rio de Janeiro, quando divididas pelas incidências correspondentes do Brasil, resultaram sempre maiores que um. A máxima relação foi observada na incidência de bacilíferos no grupo etário de zero a quatro anos, chegando a ser de 62,6 vezes, valor espantosamente alto. Como anteriormente assinalamos, é verdade que a qualidade esperada dos serviços que efetuam a baciloscopia no Rio de Janeiro seja excelente, mas verdade é também que somente qualidade de serviços não explicaria tão

\section{Tabela 6}

Coeficientes de incidência da tuberculose segundo grupo etário, formas da doença e local.

\begin{tabular}{llcc}
\hline Grupo etário (em anos) & Incidência/100.000 & Rio de Janeiro & Brasil \\
& & 1993 & 1990 \\
\hline $0-4$ & Todas as formas & 76,6 & - \\
& Forma pulmonar & 55,7 & 10,0 \\
& Baciloscopia + & 18.9 & 0,3 \\
& Meningite tb & 2,0 & 0,9 \\
$5-9$ & Todas as formas & 21,3 & - \\
& Forma pulmonar & 11,9 & 5,6 \\
& Baciloscopia + & 1,1 & 0,9 \\
$10-14$ & & & - \\
& Todas as formas & 23,8 & 4,3 \\
& Forma pulmonar & 15,2 & 3,4 \\
& Baciloscopia + & 4,47 & 8,2 \\
\hline
\end{tabular}

elevada diferença neste particular. Aí está um problema a ser pensado pelos laboratoristas/ planejadores/ epi demiologistas.

A incidência de meningite tuberculosa foi 2,2 vezes maior no Rio de Janeiro que no Brasil, e, como mostramos anteriormente, a cobertura vacinal com BCG nos últimos anos tem-se mantido sempre acima de $100 \%$. Seria erro de informação no nível do Brasil ou do Rio de Janeiro? Haveria melhor qualidade técnica disponível no Município para diagnosticar a doença? Seria cepa de BCG utilizada com baixo poder antigênico? Embora todas estas hipóteses não possam ser descartadas sem um estudo mais detalhado, quer nos parecer, contudo, que o mais provável é que as coberturas vacinais estão abarcando populações outras fora do Município e deixando parte deste a descoberto. Favoravelmente a esta hipótese, encontramos que em todas as áreas programáticas de saúde do Município, tratam sempre maior número de casos de tuberculose do que o de residentes na própria área. Este fato estaria a indicar que muitos que demandam pela assistência médica (incluindo procura por vacinação) provêm de outros municípios que não o Rio de Janeiro. De qualquer maneira, fica uma sugestão àqueles que efetuarem a vigilância da doença: procurar investigar mais detalhadamente a história de caso das meningites tubérculas.

\section{Conclusões}

Trata-se de um estudo efetuado com casos notificados e que, portanto, poderão estar subestimados; deve-se assinalar, contudo, que, nas fichas de notificação disponíveis, encontramos pelo menos $96,1 \%$ das informações que queríamos e, destas, podemos apresentar:

- o percentual relativo casos de tuberculose na infância/adultos é semelhante entre Rio de Janeiro (6,6\%) e Brasil (6,7\%); este percentual relativo é bem abaixo do valor apregoado pelo Ministério da Saúde - há fortes evidências de fatores operacionais e técnicos para sua explicação;

- setenta por cento dos casos são da forma pulmonar, seguidos da ganglionar; a distribuição segundo grupo etário segue padrão de outras regiões;

- em $50 \%$ dos casos pulmonares, foi efetuado o exame de baciloscopia, encontrando-se o (elevado) valor de $29 \%$ positivos, por razões possivelmente operacionais. Cerca de $38 \%$ dos casos pulmonares apresentavam lesões radiológicas com sinais de cavitação (evidências de diagnóstico tardio); 
- em $15,4 \%$ dos casos, foi pedida a pesquisa de anticorpos anti-HIV; uma subestimação da soroprevalência de anticorpos anti-HIV positiva neste grupo etário é de $4,1 \%$;

- o controle de comunicantes é de apenas 24\% da população alvo;

- os coeficientes de incidência de todas as formas, forma pulmonar, bacilíferos, meningite, são muitas vezes superiores (chegando a 62,6 vezes para certos coeficientes) em relação ao Brasil como um todo.

\section{Referências}

LOURES, L. A. M., 1995. Epidemiologia da interação HIV/TB. Anais do Seminário Interprogramas HIV/ Tuberculose. Brasília: Ministério da Saúde - Programa DST/AIDS.

MS (Ministério da Saúde), 1992. Controle da Tuberculose: Uma Proposta de Integração Ensino-Serviço/CNCT/NUTES. 3a ed., Rio de Janeiro: Secretaria Nacional de Programas Especiais de Saúde, Ministério da Saúde.

MS (Ministério da Saúde), 1993. Reunião de avaliação operacional e epidemiológica do programa nacional de controle da tuberculose na década de 80 . Bol etim de Pneumologia Sanitária: Número Especial. Boletim, 7:7-54.

MS (Ministério da Saúde), 1994. Programa Nacional de Doenças Sexualmente Transmissíveis/ Aids. Co-infecção TB/HIV/Aids. Brasília: Secretaria de Assistência à Saúde, Ministério da Saúde. (mimeo.)

PIO, A., 1984. El futuro de la lucha antiberculosa. Problemas y perpectivas. Boletín de la Oficina Sanitária Panamericana, 96:108-118.

RUFFINO-NETTO, A., 1995. Avaliação do excesso de casos de tuberculose atribuídos a infecção HIV/ Aids: ensaio preliminar. Revista de SaúdePública, 29:279-282.

WHO (World Health Organization), 1989. Statement on Aids and tuberculosis. Weekly Epidemiological Record, 17:125-32. 